\title{
The influence of dizocilpine on the reserpine-induced behavioral and neurobiological changes in rats
}

\section{Zhen-Yong Gao, Ping Yang, Qing-Jun Huang**, Hai-Yun Xu*}

The Mental Health Center, Shantou University Medical College, Shantou, China

${ }^{*}$ Corresponding author. Tel.: +86 75488900728

${ }^{* *}$ Corresponding author. Tel: +86 75482902704

E-mail addresses: hyxu@stu.edu.cn (H. Xu), huangqj@stumhc.cn (Q. Huang) 


\section{ABSTRACT}

Clinical studies have demonstrated that a single dose of ketamine produces complete remission within 24 hours in some depression patients. The ability of ketamine to produce fast-acting antidepressant-like effects in animal models depends on rapid synthesis of brain-derived neurotrophic factor (BDNF). Here we examined effects of a single dose dizocilpine, a non-competitive NMDA receptor antagonist, on the behavioral and neurobiological changes in rats treated with a single high dose reserpine, which is a monoamine re-uptake blocker and depletes monoamines in the brain with the outcome of depression-like symptoms in animals. A single high dose reserpine $(4.0 \mathrm{mg} / \mathrm{kg})$ was given to rats intraperitoneally. Forty-eight hours later, the rats showed depressive symptoms as evidenced by decreased locomotor activity in the open field and increased immobility time in the forced swim test. Meanwhile, the treatment decreased BDNF levels and neurogenesis in the hippocampus. Pretreatment of a single dose dizocilpine $(0.30 \mathrm{mg} / \mathrm{kg})$, however, prevented all the reserpine-induced changes, except for GluN1 subunit. These results are suggestive of the involvement of neurogenesis and BDNF in the rapid-acting antidepressant-like behavioral effects of the NMDA receptor antagonists in the reserpinized rats.

Keywords: Antidepressant; NMDA receptor; BDNF; Neurogenesis. 


\section{Introduction}

Depression is a common mental disorder affecting approximately 350 million people word-wide and resulting in decreased quality of life and major economic loss. Although the mechanisms underlying the pathogenesis and treatment of depression remain to be elucidated, significant advances have been achieved. One of these has been the monoamine theory of depression proposed by Schildkraut in 1965 [23]. This hypothesis was inspired by the clinical report on hypertensive patients who developed mental depression after treatment with high doses of reserpine [8]. Since then, extensive pharmacological studies have provided ample supporting evidence for the hypothesis, including the depletion of brain catecholamines and serotonin by reserpine-like drugs and increased availability of norepinephrine and/or serotonin by monoamine oxidase inhibitors and tricyclic drugs [20]. The monoamine hypothesis, however, does not provide an adequate explanation for observations that monoamine depletion does not produce depressive symptoms in healthy individuals and that rapid elevation in monoamines is not accompanied with a quick antidepressant action [25].

In the last decade, clinical studies have demonstrated that a single dose ketamine, a NMDA (N-methyl-D-aspartate) receptor antagonist, produces complete remission within 24 hours in some depression patients [12]. The clinical benefits after a single ketamine dose may last as briefly as 1 to 2 days or longer than 2 weeks [1]. In line with these human studies, animal studies reported antidepressant-like effects of some drugs that reduce NMDA receptor function $[13,24]$. The ability of ketamine and other NMDA receptor antagonists to produce fast-acting behavioral antidepressant-like effects in animal depends on rapid synthesis of brain-derived neurotrophic factor (BDNF) [4].

In the present study we examined effects of a single dizocilpine (MK-801, a NMDA receptor antagonist) dose on the behavioral and neurobiological changes in rats treated with 
a single high dose reserpine. On the basis of previous animal studies showing the antidepressant activity of dizocilpine [16, 18], we expected this drug would protect rats against the reserpine-induced depressive symptoms. To explore the underlying mechanisms, we measured and compared levels of BDNF and hippocampal neurogenesis in rats differentially treated with dizocilpine or/and reserpine as these two biomarkers have been demonstrated to be necessary for the fast-acting antidepressant effects in previous animal studies $[4,10,22]$. In addition, levels of GluN1 subunit of NMDA receptors in the hippocampus were assessed and compared across all animal groups. The GluN1 subunit is obligatory in the NMDA receptors and essential for the function of the receptors. It may be an important player in the pathogenesis and treatment of depression, exemplified by a recent study in which zinc-deficient rats showed depressive symptoms and higher levels of GluN1 in the hippocampus of them. Moreover, both of the changes were reversed by chronic fluoxetine administration [6]. However, it is unknown if a single high dose of reserpine or/and dizocilpine affect levels of GluN1 in the hippocampus.

\section{Materials and methods}

\subsection{Drugs}

The drugs used in this study were purchased from Sigma-Aldrich (Shanghai, China). Reserpine was dissolved in $0.05 \mathrm{~mL}$ glacial acetic acid and diluted to $2 \mathrm{mg} / \mathrm{mL}$ with distilled water. Dizocilpine was dissolved in saline at $0.15 \mathrm{mg} / \mathrm{mL}$. The thymidine analog BrdU (5-bromo-2'-deoxyuridine) was dissolved in warm saline at $10 \mathrm{mg} / \mathrm{mL}$.

\subsection{Animals and treatments}

Adult male Sprague-Dawley rats (Animal Center of Shantou University Medical College) 
weighing 220-260 g were used. They were allowed to acclimatize to the animal facilities for 7 days before the experiments. They were housed four per cage with free accesses to food and water under controlled laboratory conditions $\left(12 / 12 \mathrm{~h}\right.$ light/dark cycle, $\left.24 \pm 1^{\circ} \mathrm{C}\right)$. All the animal procedures were carried out in accordance with the guidelines set up by the Animal Care and Use Committee of Shantou University Medical College and approved by the committee.

Rats were injected intraperitoneally (i.p.) with dizocilpine $(0.30 \mathrm{mg} / \mathrm{kg})$ in saline or the same volume $(2.0 \mathrm{~mL} / \mathrm{kg})$ of saline. Thirty minutes later, rats were given (i.p.) reserpine (4.0 $\mathrm{mg} / \mathrm{kg})$ or the same volume $(2.0 \mathrm{~mL} / \mathrm{kg})$ of its vehicle. These treatments resulted in the following four groups ( $n=8 /$ group): Sal+Veh, Sal+Res, Diz+Veh, and Diz+Res. Forty-eight hours after reserpine injection, rats were subjected to behavioral tests. Immediately after the last behavioral test, rats were deeply anesthetized with $2 \%$ pentobarbital sodium and sacrificed, then the brains were removed and bilateral hippocampi were dissected and rapidly frozen and stored at $-80^{\circ} \mathrm{C}$ until ELISA analysis.

The second experiment with the same animal groups ( $n=8 /$ group) was performed. Immediately after the last behavioral test, rats were administered BrdU (75 mg/kg, i.p.) 4 times with a 2 hour interval between injections, or same volume saline. At the end, rats were anesthetized with $2 \%$ pentobarbital sodium and perfused through the ascending aorta with $0.1 \mathrm{M}$ phosphate buffered saline (PBS; $\mathrm{pH}=7.4$ ), followed by $4 \%$ paraformaldehyde in the PBS. The brains were then removed and processed to assess hippocampal neurogenesis.

\subsection{Behavioral tests}


Open field test: Locomotor activity of rats was measured by performing the open field test 48 hours after reserpine injection. Hyperactivity was associated with the dizocilpine- (2.0 $\mathrm{mg} / \mathrm{kg}$, i.p.) triggered neurotoxicity in a recent animal study [14]. We wanted to ensure the single dizocilpine $(0.3 \mathrm{mg} / \mathrm{kg}$, i.p.) dose applied here is non-neurotoxic. Briefly, individual rat was placed in the center of the open field box $(50 \times 50 \times 60 \mathrm{~cm})$ at the beginning. During the test, the rat was allowed to move freely around the open field and to explore the environment for $5 \mathrm{~min}$. The paths of the animal were recorded by a video camera above the arena and analyzed by a video tracking system (DigBehav system,yishu Co., Ltd). The arena was carefully cleaned with $70 \%$ alcohol and rinsed with water after each test.

Forced swimming test: The forced swimming test was conducted 30 min after the openfield test. It involves two individual exposures to a cylindrical tank $(65 \mathrm{~cm}$ high $\times 30 \mathrm{~cm}$ diameter) with water $\left(25^{\circ} \mathrm{C}\right)$ in which rats cannot touch the bottom of the tank or escape. Water in the tank was changed after each rat-test. First, a rat was placed in the water for 15 min (pre-test session). Twenty-four hours later, the rat was placed in the water for 5 min (test session). The immobility time of the rat was recorded. It was considered to be immobile when a rat remained floating in the water without struggling or making only minimum movements necessary for keeping his head above the water.

\subsection{Enzyme-linked immunosorbent assay (ELISA)}

NMDA GluN1 and BDNF levels in the hippocampus were measured by ELISA method. The kits were available commercially (GluN1 kit, CUSABIO, Wuhan; BDNF kit, BOSTER, Wuhan, China). Briefly, the hippocampus tissue was homogenized in lysis buffer (50 mM 
Tris $\mathrm{pH}$ 7.4, $150 \mathrm{mM} \mathrm{NaCl}, 4 \mathrm{mM}$ EDTA, 1\% sodium azide, 1\% Triton-X100, 1mM EGTA and $1 \mathrm{mM}$ phenylmethylsulfonyl fluoride (PMSF)) of 10 volumes of the wet tissue. The homogenate was centrifuged at $14,000 \times g$ for 5 minutes at $4^{\circ} \mathrm{C}$, then the supernatant was removed and the amount of GluN1 and BDNF protein in each sample was measured in duplicate according to the manufacture's protocol.

\subsection{BrdU immunohistochemistry}

The frozen brain tissue was sectioned $(40 \mu \mathrm{m})$ in the coronal direction using the cryostat. Then, the brain sections were incubated in $30 \% \mathrm{H}_{2} \mathrm{O}_{2}$ in methanol (1:50) for 30 min at $24{ }^{\circ} \mathrm{C}$ to block endogenous peroxidase. After PBS rinses, sections were incubated in $0.1 \%$ Triton $\mathrm{X}-100$ for $10 \mathrm{~min}$ at $4^{\circ} \mathrm{C}$ and then denatured by incubation in $2 \mathrm{M}$ hydrochloric acid for $30 \mathrm{~min}$ at $37^{\circ} \mathrm{C}$. Following washing, sections were preincubated for $20 \mathrm{~min}$ in $5 \%$ BSA at $24^{\circ} \mathrm{C}$, followed by incubation with primary mouse monoclonal anti-BrdU antibody (1:100; Ebio Science, USA) at $4^{\circ} \mathrm{C}$ overnight. The sections were then washed and incubated with horseradish peroxidase (HRP)-labeled anti-mouse IgG with amino acid polymer (Boster; Wuhan, China) for $30 \mathrm{~min}$ at $37^{\circ} \mathrm{C}$. After PBS rinses three times, sections were stained with DAB solution (Boster; Wuhan, China), counterstained with hematoxylin, dehydrated in sequential ethanol, and transparent with dimethyl benzene in the order. Finally, brain sections were sealed with neutral gum prior and observed under a Nikon H550L microscope (Nikon Corporation, Japan). Immunohistochemical controls were performed as above except for the omission of the primary antibodies. 


\subsection{Image analysis}

Digital images were acquired using the Nikon H550L microscope and the numbers of BrdU positive cells in the hippocampus were counted using Image-Pro Plus Version 6.0 color image analysis system. Final results were summarized from three visions of each section with the hippocampus, six sections of each rat, and five rats of each group. The results were expressed as BrdU positive cell number per section.

\subsection{Statistical analysis}

Results were expressed as mean \pm SEM. The data were analyzed by two-way ANOVA and followed by LSD test or Dunnett's T3 test (if variances are irregularity) for post-hoc comparisons. A $P$ value of less than 0.05 was regarded as statistically significant.

\section{Results}

\subsection{Dizocilpine prevented the reserpine-induced depressive symptoms}

In the open field, rats in the Sal+Res group traveled a shortest distance. In details, two-way ANOVA showed that the interaction between reserpine and dizocilpine $\left[F_{(1,60)}=\right.$ 3.209, $P=0.078$ ] failed to reach significance in terms of the total distance traveled in the open field. Dizocilpine had no effect $\left[F_{(1,60)}=1.731, P=0.193\right]$, although reserpine produced a significant effect $\left[\mathrm{F}_{(1,60)}=39.275, P<0.001\right]$. Given the interaction is close to significant, post hoc comparisons were performed, which revealed a significant decrease in the total distance of the Sal+Res group compared with the Sal+Veh group $(P<0.001$, Fig. $1 \mathrm{~A})$ and a significant increase in this parameter of the Diz+Res group compared with the Sal+Res group $(P<0.050$, Fig. 1A). For the distances traveled in the central area (Fig. 1B) of the 
open field, the interaction between the two factors was significant $\left[F_{(1,60)}=7.392, P=0.009\right]$. Post hoc comparisons revealed significant differences between Sal+Veh and Sal+Res $(P<$ 0.001), and between Sal+Res and Diz+Res $(P<0.01)$. For the ratio CD/TD (Fig 1C), the interaction between the two factors was significant $\left[F_{(1,60)}=7.790, P=0.007\right]$. The difference between Sal+Veh and Sal+Res was significant $(P<0.05)$, and so was it between Sal+Res and Diz+Res $(P<0.01)$.

In the forced swimming test, rats in the Sal+Res group presented a longest immobility time. In details, two-way ANOVA showed a significant interaction between reserpine and dizocilpine $\left[\mathrm{F}_{(1,60)}=97.043, P<0.001\right]$. Both reserpine $\left[\mathrm{F}_{(1,60)}=85.121, P<0.001\right]$ and dizocilpine $\left[F_{(1,60)}=113.195, P<0.001\right]$ produced significant effects on the immobility time in the water tank. Post hoc comparisons revealed a significant increase of immobility time in the Sal+Res group compared with the Sal+Veh group $(P<0.001$, Fig. 1D) and a significant decrease of immobility time in the Diz+Res group compared with the Sal+Res group $(P<$ 0.001, Fig. 1D).

3.2. Dizocilpine blocked the reserpine-induced decrease in BDNF, but had no effect on levels of GluN1 subunit in the hippocampus

The levels of BDNF in the hippocampus tissue of rats were quantified by means of ELISA. Statistical analyses showed that the interaction between dizocilpine and reserpine $\left[\mathrm{F}_{(1,28)}=3.029, P=0.093\right]$ failed to reach significance. Given the interaction is close to significant, post hoc comparisons were performed, which indicated that the level of BDNF in the Sal+Res was significantly decreased compared with the Sal+Veh group $(P<0.001$, Fig. 2A). But, no difference was found between the Sal+Veh and Diz+Res groups, the level of 
BDNF in the latter group was significantly higher compared with the Sal+Res group $(P<$ 0.001, Fig. 2A).

We also measured levels of GluN1 subunit in the hippocampus tissue of rats. Statistical analyses (Fig. 2B) showed no interaction between reserpine and dizocilpine $\left[F_{(1,28)}=0.655\right.$, $P=0.425]$ as dizocilpine had no effect on the level of GluN1 $\left[\mathrm{F}_{(1,28)}=3.524, P=0.071\right]$, whereas reserpine showed a significant effect $\left[F_{(1,28)}=25.779, P<0.001\right]$.

\subsection{Dizocilpine blocked the reserpine-induced decrease of the number of BrDU positive} cell in the dentate gyrus

The number of BrdU positive cell in the dentate gyrus of rats was quantified and compared across all four groups (Fig. 3A). The statistical analysis showed a significant interaction between reserpine and dizocilpine $\left[\mathrm{F}_{(1,28)}=13.820, P=0.001\right]$. Both reserpine $[F(1,28)=15.964, P<0.001]$ and dizocilpine $\left[\mathrm{F}_{(1,28)}=6.433, P=0.017\right]$ showed significant effects on the number of BrdU positive cell. Post-hoc comparisons revealed a significant decrease of BrdU positive cell in the Sal+Res group compared with the Sal+Veh group $(\mathrm{P}<0.01$, Fig.3B), and a significant increase of BrdU positive cell in the Diz+Res group compared with the Sal+Res group $(P<0.01$,Fig.3B).

\section{Discussion}

Reserpine is a monoamine re-uptake blocker which depletes monoamines in the brain. It has been extensively used in animal studies to produce parkinsonian symptoms $[7,17]$ and mimic depressive symptoms [2, 3, 9]. Of the depression animal models, repeated administrations of low doses reserpine in consecutive days have been successfully 
employed [2, 3]. A single dose of $2 \mathrm{mg} / \mathrm{kg}$ (i.p.) [9], but not $0.2 \mathrm{mg} / \mathrm{kg}$ (i.p.) [2], reserpine induced depressive symptoms. The reserpine-induced depressive symptoms were accompanied with significant decreases in levels of monoamines including dopamine (DA), norepinephrine (NE) and serotonin (5-HT) in certain brain regions [2, 3, 9]. In accordance with these previous studies, a single dose of $4.0 \mathrm{mg} / \mathrm{kg}$ reserpine in the present study induced depressive symptoms in rats as evidenced by decreased locomotor activity of reserpinized rats in the open field and increased immobility time in the water tank. These results, together with those of the abovementioned previous studies, suggest that the diminishment of monoamines in the brain to a certain level is essential for the induction of depressive symptoms in rodents, either by a single high dose or repeated administrations of a low dose reserpine.

The reserpine-induced depressive symptoms were blocked in rats pretreated with a single dizocilpine dose, suggesting a rapid-acting antidepressant-like activity of this NMDA antagonist. The dizocilpine alone did not affect the locomotoactivity of rats, sugggesting that the single low dose of this drug had no neurotoxicity in the present study. We did not measure levels of monoamines in brains of rats thus were unable claim that the antidepressant-like activity of dizocilpine shown here was due to an upregulation of brain monoamines by this NMDA antagonist, although previous studies have reported that dizocilpine and phencyclidine increased levels of endogenous DA, either through stimulation of synthesis and release or blockade of reuptake [21]

Reserpinized rats showed higher levels of GluN1 in the hippocampus while they displayed depressive symptoms compared to controls, suggesting the involvement of increased GluN1 in the behavioral changes. In line with this suggestion, a recent study reported an increase in GluN1 in the hippocampus of zinc-deficient rats, which also showed depressive symptoms. More interestingly, the both changes were reversed by chronic 
fluoxetine administration [6]. Similarly, reduced amygdala-hypothalamic protein levels of GluN1-containing NMDA complexes were associated with the depression-like behaviors elicited by dietary magnesium restriction. But chronic paroxetine treatment did not normalize the altered protein levels whereas it improved depression-like behaviors in the magnesium restriction mice [11]. A single dizocilpine dose blocked the reserpine-induced depressive symptoms, but had no effect on the increased levels of GluN1 subunit in the present study, suggesting that this drug targeted the downstream of the NMDA receptor, rather than the receptor per se, as paroxetine did.

The implication of BDNF in the rapidly acting antidepressant-like behavioral effects of some NMDA receptor antagonists was elegantly demonstrated in a recent study, in which ketamine and dizocilpine significantly reduced immobility time of wild-type mice in water tank, but had no effect in inducible BDNF knockout mice [4]. In the present study, a single dizocilpine dose improved the depressive symptoms of reserpinized rats and blocked the BDNF decrease in the hippocampus of them. The results confirmed the dependence of rapid-acting antidepressant-like activity on BDNF as demonstrated in the previous study [4] and extended this conclusion to the reserpinized rats, another animal model of depression.

Hippocampal neurogenesis has been believed to be an important player in the pathophysiology and treatment of depression. Animal studies have provided evidence that hippocampal neurogenesis is negatively regulated by stress and positively regulated by antidepressant treatment [15]. For the first time, the present study showed that a single reserpine dose decreased cell proliferation in the hippocampal dentate gyrus of rats while the same treatment induced depressive symptoms in the subjects. More significantly, this reserpine-induced decrease in hippocampal cell proliferation was blocked by a single dose of dizocilpine. These results suggest that hippocampal neurogenesis may be involved in the antidepressant-like behavioral effects of dizocilpine. This suggestion is in line with the 
currently accepted notion that hippocampal neurogenesis may be on the final step of the common pathway leading to antidepressant effects. Upstreams to hippocampal neurogenesis include BDNF upregulation and modulation of NMDA receptors as shown in the present and previous study showing the inhibition of NMDA receptor signaling by dizocilpine increased adult neurogenesis [19] .

In summary, a single high dose reserpine induced depressive symptoms in rats. Meanwhile, it decreased hippocampal BDNF levels and hippocampal neurogenesis. Pretreatment of a single dose dizocilpine blocked all the reserpine-induced changes. This study provided a simple animal model able to link monoamines depleting to depressive symptoms and to test the involvement of BDNF in the rapid-acting antidepressant-like behavioral effects of NMDA receptor antagonists.

Of the limitations, this study did not measure levels of monoamines in brains of rats. And it is difficult to say that the abnormal performances of the reserpinized rats in open-field fest were indicative of depressed symptoms, rather than Parkinson's-like behaviors. Future study should perform repeated low-dose reserpine treatment.

\section{Funding}

This study was supported by the National Science Foundation of China (30770771). 


\section{References}

[1] M. Aan Het Rot, C.A. Zarate Jr, D.S. Charney, S.J. Mathew, Ketamine for depression: Where do we go from here? Biol. Psychiatry 72 (2012):537-547.

[2] L. Antkiewicz-Michaluk, A. Wasik, E. Mozdzen, I. Romanska, J. Michaluk, Antidepressant-like effect of tetrahydroisoquinoline amines in the animal model of depressive disorder induced by repeated administration of a low dose of reserpine: behavioral and neurochemical studies in the rat, Neurotox. Res. 26 (2014): 85-98.

[3] V. Arora, A. Kuhad, V. Tiwari, K. Chopra, Curcumin ameliorates reserpine-induced pain-epression dyad: Behavioural, biochemical, neurochemical and molecular evidences, Psychoneuroendocrinology 36 (2011): 1570—1581.

[4] A.E. Autry, M. Adachi, E. Nosyreva, E.S. Na, M.F. Los, P.F. Cheng, et al., NMDA receptor blockade at rest triggers rapid behavioural antidepressant responses, Nature 475 (2012): 91-95.

[5] W. Danysz, C.G. Parsons, J. Kornhuber, W.J. Schmidt, G. Quack, Aminoadamantanes as NMDA receptor antagonists and antiparkinsonian agents—preclinical studies. Neurosci. Biobehav. Rev. 21(1997): 455-468.

[6] U. Doboszewska, B. Szewczyk, M. Sowa-Ku'cma, K. M, A. Rafało, B. Ostachowicz, M. Lankosz, et al., Antidepressant activity of fluoxetine in the zinc deficiency model in rats involves the NMDA receptor complex, Behav. Brain Res. 287 (2015):323-330.

[7] R. C. Dutra, A. P. Andreazza, R. Andreatini, S. Tufik, M. A.B.F. Vital, Behavioral effects of MK-801 on reserpine-treated mice, Prog. Neuropsychopharmacol. Biol. Psychiatry 26 (2002): 487-495.

[8] E.D. Freis, Mental depression in hypertensive patients treated for long periods with large doses or reserpine, N. Engl. J. Med. 251 (1954): 1006-1008. 

antidepressant-like effects in animal models: role of brain-derived neurotrophic factor, Behav. Brain Res. 238 (2013): 79-85.

[10] L.S. Garcia, C. M. Comim, S. S. Valvassori, G. Z.Reus, L. M. Barbosa, A. C. Andreazza, et al., Acute adminis-tration of ketamine induces antidepressant-like effects in the forced swimmingtest and increases BDNF levels in the rat hippocampus, Prog. Neuropsychophar-macol. Biol. Psychiatry 32 (2008): 140-144.

[11] M. Ghafari, N. Whittle, A. Miklo'si, C. Kotlowsky, C. Schmuckermair, J. Berger, et al., Dietary magnesium restriction reduces amygdala-hypothalamic GluN1 receptor complex levels in mice, Brain Struct. Funct. DOI 10.1007/s00429-014-0779-8.

[12] E.T. Kavalali, L. M. Monteggia, How does ketamine elicit a rapid antidepressant response? Cur. Opin. Pharmacol. 20 (2015):35-39.

[13] R.T. Layer, P. Popik, T. Olds, P. Skolnick, Antidepressant-like actions of the polyamine site NMDA antagonist, eliprodil (SL-82.0715), Pharmacol. Biochem. Behav. 52 (1995): $621-627$.

[14] J. M. Lima-Ojeda, M.A. Vogt, N. Pfeiffer, C. Dormann, G. Kohr, R. Sprengel, et al., Pharmacological blockade of GluN2B-containing NMDA receptors induces antidepressant-like effects lacking psychotomimetic action and neurotoxicity in the perinatal and adult rodent brain. Prog Neuropsychopharmacol Biol Psychiatry 45 (2013): 28-33.

[15] I. Mahar, F. R. Bambico, N. Mechawar, J. N. Nobrega, Stress, serotonin, and hippocampal neurogenesis in relation to depression and antidepressant effects, Neurosci. Biobehav. Rev. 38 (2014):173-192. 
[16] J. Maj, Z. Rogoz, G. Skuza, H. Sowinska, Effects of MK-801 and antidepressant drugs in the forced swimming test in rats, Eur. Neuropsychopharmacol. 2 (1992): 37-41.

[17] J. E. Nash, M. P. Hill, J.M. Brotchie, Antiparkinsonian actions of blockade of NR2B-containing NMDA receptors in the reserpine-treated rat, Exp. Neurol. 155(1999): 42-48.

[18] M. Papp, E. Moryl, New evidence for the antidepressant activity of MK-801, a non-competitive antagonist of NMDA receptors, Pol. J. Pharmacol. 45(1993): 549-553.

[19] D. S. Petrus, K. Fabel,G. Kronenberg, C. Winter, B. Steiner. G. Kempermann, NMDA and benzodiazepine receptors have synergistic and antagonistic effects on precursor cells in adult hippocampal neurogenesis. Eur. J. Neurosci. 29 (2009): 244-252.

[20] J.C. Pryor, F. Sulser, Evolution of the monoamine hypothesis of depression. In: Horton RW, Katona CLE, editors. Biological aspects of affective disorders. New York: Academic Press; 1991. P.77-94.

[21] M.G. Richard, J.P. Bennett Jr., NMDA receptor blockade increases in vivo striatal dopamine synthesis and release in rats and mice with incomplete, dopamine-depleting, nigrostriatal lesions, J. Neurochem. 64(1994): 2080-2086.

[22] L. Santarelli, M. Saxe, C. Gross, A. Surget, F. Battaglia, S. Dulawa, et al., Requirement of hippocampal neurogenesis for the behavioral effects of antidepressants, Science 301(2003):805-809.

[23] J.J. Schildkraut, The catecholamine hypothesis of affective disorders: a review of supporting evidence. Am. J. Psychiatry 122(1965):509-522.

[24] R. Trullas, P. Skolnick, Functional antagonists at the NMDA receptor complex exhibit antidepressant actions, Eur. J. Pharmacol. 185(1990):1-10. 
[25] V.A. Vaidya, R.S. Duman, Depression-emerging insights from neurobiology, Br. Med. Bull. 57 (2001):61-79. 


\section{Figure legends}

Fig. 1. Dizocilpine ameliorated the depressive symptoms in the reserpinized rats. (A) Rats in the Sal+Res group traveled a shortest distance in the open field, but this reserpine-induced change was ameliorated in the Diz+Res group. (B) Rats in the Sal+Res group traveled a shortest distance in the central area of the open field, but it was ameliorated in the Diz+Res group. (C) Rats in the Sal+Res group showed a smallest ratio of CD/TD, but it was blocked in the Diz+Res group. (D) Rats in the Sal+Res group showed a longest immobility period in the forced swim test, but it was blocked in the Diz+Res group. Data are expressed as mean $\pm S . E . M\left(n=16 /\right.$ group). ${ }^{*} p<0.05,{ }^{* *} p<0.01,{ }^{* * *} p<0.001$, compared to Sal+Veh; $+p<0.05$, $++p<0.01,+++p<0.001$, compared to Sal + Res.

Fig. 2. Dizocilpine blocked the reserpine-induced decreases in levels of BDNF, but had no effect on the GluN1 subunit. (A) Rats in the Sal+Res group showed a lowest level of hippocampal BDNF, but it was blocked in the Diz+Res group. (B) all reserpinized rats showed higher levels of GluN1 subunit protein in the hippocampus. Data are expressed as mean $\pm S . E . M\left(n=8 /\right.$ group). ${ }^{* * *} p<0.001$, compared to Sal+Veh; $+++p<0.001$, compared to Sal+Res.

Fig. 3. Dizocilpine blocked the reserpine-induced decreases in BrdU positive cells. (A) Representative microphotographs showing BrdU positive cells in the dentate gyrus of rat. (B) Rats in the Sal+Res group showed a lowest number of BrdU positive cells, but it was blocked in the Diz+Res group. Data are expressed as mean \pm S.E.M $\left(n=8 /\right.$ group). ${ }^{* *} p<0.01$, compared to Sal+Veh; ++p<0.01, compared to Sal+Res. 

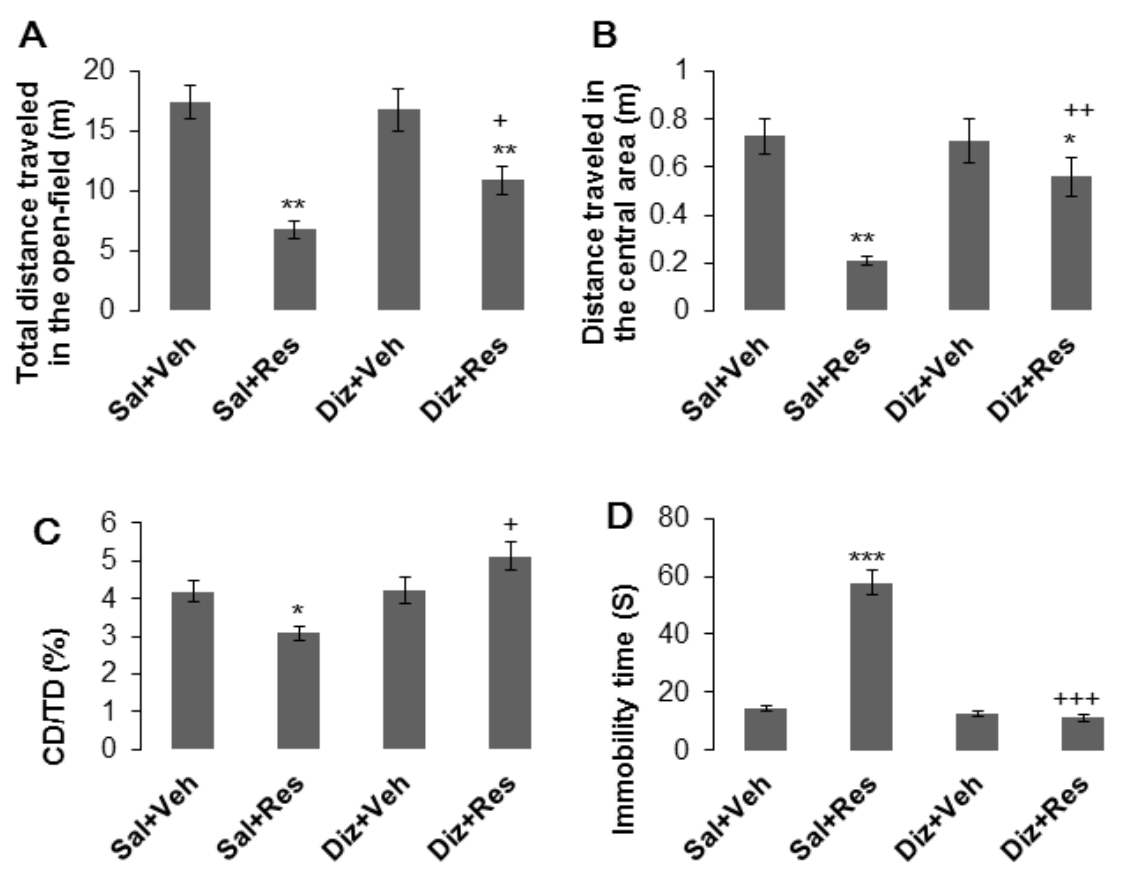


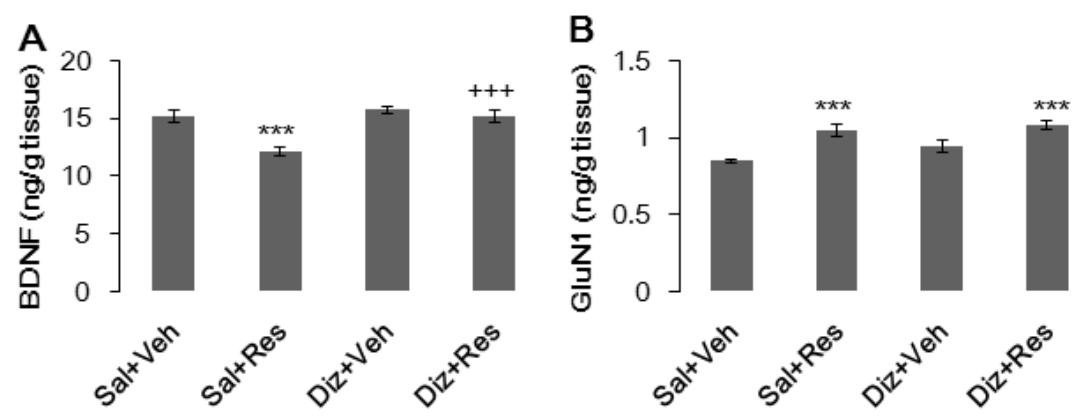



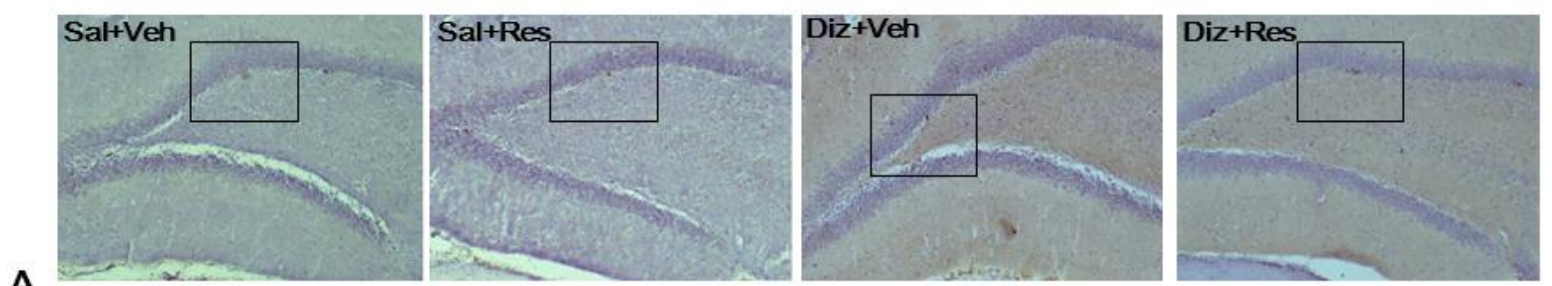

A
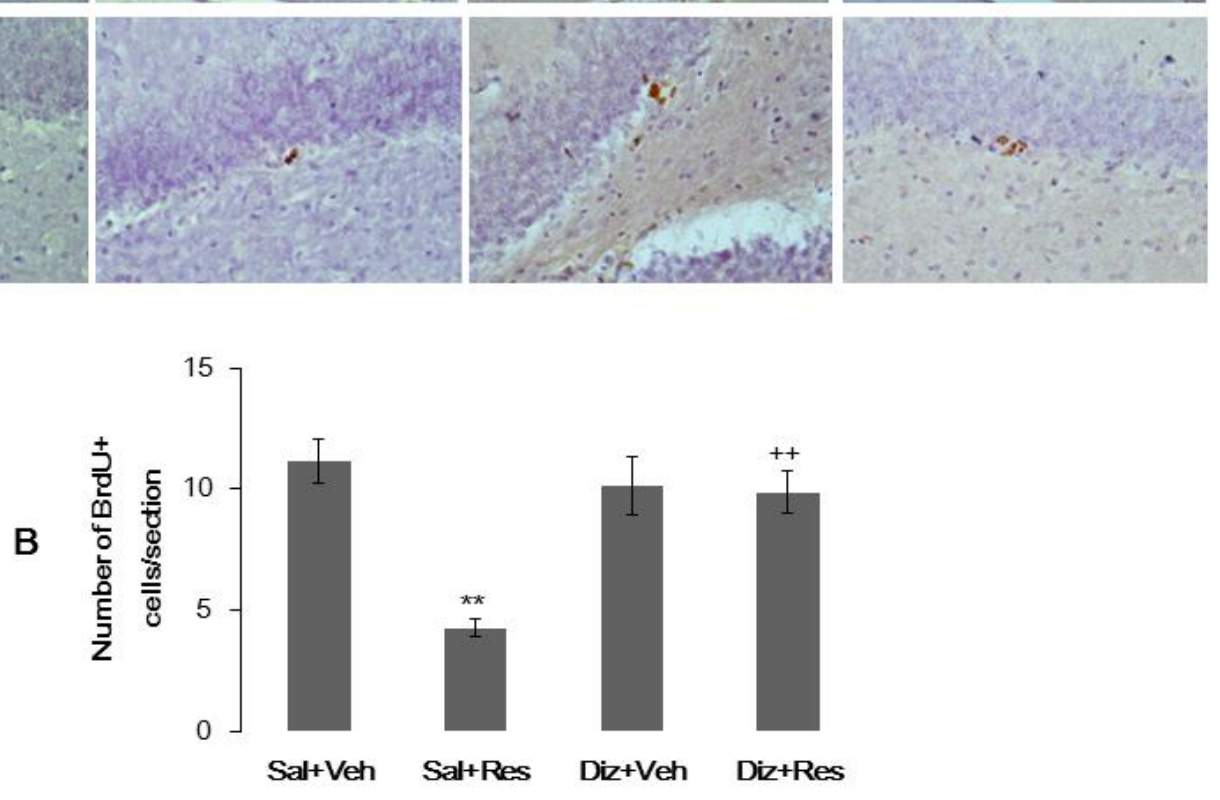